\title{
Pesquisas sobre a physiologia do lobo anterior da hypophyse e das glandulas sexuaes
}

Pelo Dr. THALES MARTINS

(Assistente do Instituto Oswaldo Cruz).

Os trabalhos recentes de ZONDEK e ASCHHEIM (1), SMITH e ENGLE (2) estabeleceram seguramente o papel do lobo anterior da hypophyse como regulador das glandulas sexuaes. E' sabido que a implantação do lobo anterior da pituitaria, ou as injecções de determinados extractos desse orgão, provocam nos animaes infentis, machos ou femeas, a puberdade precoce. O sexo, a especie ou a idade do animal doador não importam no resultado, qual itativamente, pelo menos entre os mammiferos.

As hypophyses de aves não dão resultados completos, (SMITH e ENGLE 2); LIPSCHÜTZ, KALLAS e WILKENS (3a), e assim tambem as de rans chilenas (Kalyptocephalus) durante o repouso sexual (LIPSCHÜTZ e PAEZ, (3b), ou mesmo em periodo de cio, (TH. MARTINS, 4) nas rans brasileiras Leptodactyllus. Em toda a serie de trabalhos ahi referidos, trata-se de implantações feitas em roedores infantis. Entretanto, HOUSSAY e GONZALEZ (5) observaram hypertrophia testicular nos sapos normaes ou hypophyseoprivos, após implantaçoes de hypophyses de sapo.

Podemos considerar o papel estimulador da prehypophyse como geral nos mammiferos; nestes, resultados similares têm sido obtidos em grupos zoologicos os mais diversos, e até mesmo em primatas (ALLEN, 6).

A rapidez e a segurança com que os ovarios dos pequenos roedores reagem á prepituitaria, fez delles um test coınmodo para pesquisas no assumpto, e permittiu um grande numero de verificações interessantes. Assim, ZONDEK e ASCHHEIM (7) demonstraram a enorme eliminação de hormonio do lobo anterior durante a gravidez, ao lado do hormonio ovariano; é tão constante esse facto, que a pesquisa delle na urina constitue hoje o melhor e o mais precoce processo de diagnostico da gravidez, na especie humana. Facto digno de nota é que, alem do homem, só no chimpanzé elle foi verificado.

\section{PUBERDADE E ELIMINAÇÃO DE HORMONIOS HYPOPHYSIARIOS.}

Na puberdade masculina o hormonio do lobo anterior pode ser encontrado na urina, como referem recentemente DE JONG, LAQUEUR e DINGEMANSE (8), em resumo.

Tambem verificamos esse facto na puberdade feminina; fizemos a pesquiza em 6 moçoilas, quando se iniciavam os primeiros symptomas menstruaes. Em 2, foi possivel demonstrar a existencia de hormonios prehypophysarios na urina, pela provocação da puberdade precoce nas camon. dongas infantis (pesando 6 a 7 grs.), injectadas. Ahi, os factos positivos 
são decisivos, e os negativos não têm grande importancia, porque não se sabe qual o momento preciso em que se dá a eliminação; isso se poderá fazer facilmente, pela pesquiza systematica da urina, acompanhando as pacientes desde um praso mais ou menos longo, antecedente á puberdade.

\section{HVPOPHYSE E LEI DO “TUDO OU NADA".}

PÉZARD (9) estabeleceu a lei do tudo ou nada, para o funccionamento endocrino do testiculo, nos gallinaceos. Elle demonstrou que acima de uma quantidade minima de testiculo, os animaes são normaes, no ponto de vista dos caracteres sexuaes. Abaixo desse minimo efficaz, o animal é igual a um castrado total. Não é possivel obter iypos intermediarios, á custa de uma reduccão da massa testicular. LIPSCHÜTZ e seus collaboboradores (10) demonstram que nas cobayas basta $1 \%$ do peso normal de testiculo para manter o penis e glandulas annexas em nivel normal.

PEZARD estendeu aos ovarios a lei em questão; os novos conhecimentos sobre o hormonio ovariano trazem fortes restricções a isso; mas quanto ao testiculo, nenhum argumento de valor foi opposto. Todavia, as noções recentes sobre o papel do lobo anterior da hypophyse exijiam uma nova verificação da lei, principalmente quanto á possibilidade de provocar uma hyperfuncção testicular.

De facto, estabelecido um factor de regulação, comprehende-se muito bem que, com o enxerto de varios testiculos em animal normal, nada se obtenha de supra-normal, como verificou PEZARD. E ainda que a reducção de massa seja compensada por uma hyper-secreção, apezar de histologicamente, não serem encontrados no pequeno fragmento restante sig$\mathrm{n}$ aes seguros de hyper-funcção.

Actualmente, a lei do "tudo ou nada" poderia ser interpretada de trez modos:

10-A hypophyse funcciona "tudo or nada", isto é, ou não estimula, ou provoca no testiculo reacções maximaes.

20-O testiculo só reage tudo ou nada, quer dizer, a partir de uma certa quantidade de hormonio hypophysario elle daria o maximo de effeito; ás concentrações maiores de hormo nio hyp. elle seria indifferente.

3o-A lei do tudo ou nada r eside nos orgãos receptores, isto é, crista dos gallinaceos, penis, glandulas annexas, etc., nos mammiferos.

Com as implantações da hypophyse ou injecção de material activo, podemos induzir no animal um estado de hyper-hypophysismo.

SMITH e ENGLE implantaram diariamente pituitaria anterior, por um periodo de 15 a 35 dias, em ratos adultos. Não houve augmento de peso nem alterações estructuraes na genitalia dos animaes em experiencia.

Nós fizemos 3 a 4 implantaçð̃es de lobo anterior de hyp. de coelho em camondongos de $18-20$ grs., durante um praso de 60 a 90 dias. Ao fim desse tempo, o tracto genital era examinado. Tomamos como guia as dimensões das vesiculas seminaes, e o peso dos testiculos. Em 4 animaes de 18-20 grs., as dimensões foram $14,5 \times 5 \mathrm{~mm}$. (comprimento e largura maximas) $; 15 \times 5 ; 20 \times 4,5$; numeros que estão dentro dos limites normaes. O que nos chamamos "indice dimensional das vesiculas" (11), isto é, o producto $\mathrm{L} \times \mathrm{H}$, seria, nos animaes acima, respectivamente $72,5,75$ e 90 . Nos animaes normaes o indice varia de 60 a 115 . 
O peso dos testiculos não é superior ao normal. Injectamos então hormonio hypophysario extrahido da urina de nulheres gravidas, isento de hormonio ovariano e previamente verificado activo em animaes infantis. Ainda assim, nada de positivo pudemos obter, injectando 3 a 4 vezes por semana cerca de 5 a 10 unidades ZONDEK-ASCHHEIM, de cada vez; a experiencia foi feita em 4 cammondongos de $20-23$ grs.

Histologicamente, não encontramos nada no testiculo que pudesse traduzir um super actividade. Apenas, em um caso de animal cryptorchidico, o testiculo abdominal apresentava uma hypertrophia intersticial que excedia muito a que se observa de regra nessa situação. Quer na nossa colleção de testiculos cryptorchidicos, quer nas microphotographias encontradas em trabalhos de diversos autores, nenhum apresenta tanta riqueza de elementos intersticiaes. E' um facto que merece elucidação posterior, tanto mais quanto no testiculo normalmente descido nenhuma reacção intersticial foi observada, nos animaes injectados ou implantados.

Em conclusão, com a technica descripta não foi possivel induzir nos orgâos sexuaes secundarias nenhum desenvolvimento acima do normal, que traduzisse uma hyperfuncção testicular.

Somente o emprego de um extracto testicular activo e sufficientemente puro para poder ser injectado em quantidades crescentes, resolverá a questão.

Quando se puder ter segurança de que realmente um animal com excesso de hormonios testiculares em circulação não ultrapassa a morphologia sexual normal, então será possivel determinar até que ponto vae a capacidade de reacção nos orgãos receptores.

\section{CASTRAÇÃO E HYPOPHYSE.}

E' sabido que após a castração, a hypophyse se hypertrophia, e modificações histologicas se estabelecem no lobo anterior (FICHERA 12).

As hypophyses dos animaes castrados, implantadas em ratos infantis, mostram actividade maior que as retiradas de animaes normdes (ENGLE, 13, EVANS e SIMPSON 14).

$\mathrm{Na}$ ausencia das gonadas, portanto, ha um enriquecimento de hormonio na glandula. Esse facto poderia ser interpretado por um accumulo de hormonios, que não seriam secretados após a castração. Mas ha evidencias de que depois da ablação das gonadas elles circulam em quantidade dosavel.

MATSUYAMA (15) publicou ha annos um trabalho de grande valor e que não exerceu entretanto, grande influencia sobre a marcha das pesquisas em torno das glandulas sexuaes

Elle uniu em parabiose ratos normaes e castrados. Pela união de ratos normaes do mesmo sexo, não ha modificaçðes genitaes que nos interessem. Mas a ligação de um castrado com um normal tem consequencias da maior importancia. E' assim que a atrophia do utero, ou do penis e glandulas annexas do companheiro castrado não se modificam com a união a uma femea normal; mas a genitalia desta soffre modificações consideraveis. Os ovarios crescem enormemente; tornam-se cysticos, e depois ricamente luteinisados. O utero atinge proporçð̃es enormes; ha hypertrophia e hypersecreção, que o distende. 
GOTO (16) repetiu e confirmou as experiencias de MATSUYAMA.

MATSUYAMA não deixa transparecer qual a interpretação desses phenomenos; GOTO admitte a existencia de um "Kastrhormon", substancia secretada só nos animaes castrados; com a injecção do sangue destes, provoca alterações genitaes em femeas normaes adultas.

Os conhecimentos actuaes permittem lançar um pouco de luz sobre esses phenomenos insolitos.

A interpretação mais simples e mais logica é a de que se trata do hormonio do lobo anterior da hypophyse, que passa do animal castrado para o animal normal, em parabiose.

KALLAS (17) uniu em parabiose ratos infantis; femeâs castradas com femeas normaes. No fim de alguns dias, os ultimos entram em puberdade precoce, traduzida pela maturação do ovario, abertura da vagina e cio.

KALLAS é o primeiro autor que evidencia a possibilidade de ser identificada com o hormonio do lobo anterior a substancia circulante no castrado, o "Kastrhormon" de GOTO; e ainda vê na experiencia uma demonstração de que mesmo nos animaes infantis o hormonio do lobo anterior existe em circulação.

Estamos de accordo quanto á identidade entre a substancia em questão e o hormonio do lobo anterior. Discordamos, entretanto, que a experiencia prove que nos animaes infantis normaes os hormonios referidos já circulem.

Poder-se-ia julgar que, na ausencia dos ovarios, isto é, dos orgãos que normalmente fixam o hormonio hypophysario, esse ficasse livre na circulação e passasse do castrado para o normal. Mas no caso dos infantis, a concentração delles no sangue está evidentemente abaixo do limiar necessario á provocação da puberdade; e a pequena quantidade que passa de um para outro, torna difficil explicar a constancia dos effeitos, isto é, a puberdade num rato de 15 a 20 grs., quando normalmente ella só se dá em animaes pesando mais de 50 grs.

As injecções de sangue ou soro sanguineo por nós realisadas tambem demonstram a presença de hormonio hypophysario circulante nos castrados.

\section{PUBERDADE PRECOCE PELA INJEÇÃO DE SANGUE DE ANIMAL CASTRADO.}

Ratos infantis machos e femeas foram castrados, com um peso de 25-30 grs. 8 dias após, o sangue retirado por puncção do coração era injectado em ratos infantis daquelle peso. A injecção 2 a 3 vezes por dia, de 0,20 a 0,3 de sangue ou de soro, provocaram no animal injectado a abertura da vagina e o estro, a partir do 50 dia. No ovario, encontram-se os folliculos desenvolvidos e, mais raramente, corpora lutea atresica. A injecção de sangue nas mesmas condições, retirado de animaes infantis normaes, não provoca esses phenomenos, mesmo injectando doses maiores.

Dir-se-a que nos animaes infantis ou adultos, os ovarios fixem o hormonio circulante. Mas o sangue retirado em dado momento do coração, não passou todo pelo ovario.

A interpretação que nos parece rasoavel é a de que na castração 
haja uma hvperfuncção de hypophyse; as gonadas exercem uma influencia directa ou indirecta sobre a a pituitaria, existindo portanto entre as duas glandulas uma interrelação mutua.

Dos factos acima descriptos, podemos por em evidencia que, na castração, observa-se em relação á hypophyse: 10 Hypertrophia. 20 Enriquecimento da glandula em hormonios. 3o Existencia delles em circulação, em grande quantidade.

A gravidez nos primatas é ainda um exemplo de influencia da genitalia sobre a hypophyse; si não foi ainda determinado de onde parte o estimulo para a enorme descarga de hormonios que se observa nessa situação, é obvio que primariamente na genitalia elle deve estar localisado; e a partir de certo tempo, talvez na placenta.

Para decidir a questão do funccionamento precoce da hypophyse ou da sua hyperfuncção, após a castração, temos experiencias em curso, nas quaes a hypophyse de um ou de ambos os animaes em parabiose, foi retirada.

\section{O CY CLO ESTRAL DOS ANIMAES EM PARABIOSE.}

Nas experiencias de MATSUYAMA e de GOTO, a atrophia do utero dos animaes castrados não se modificava quando elles eram unidos em parabiose, com animaes normaes. Pareceria, portanto, que o hormonio ovariano não passa de um para outro. Isso seria explicavel pela presença do ut ero, vagina ou outros fixadores eventuaes daquelle hormonio; desse modo o hormonio secretado nos ovarios seria todo elle consumido no organismo do animal normal.

Nós nos propuzemos a estudar o cyclo estral dos animaes em parabiose; para essa ultima questão, unimos uma femea castrada a uma normal, da qual retiramos completamente o utero.

Mas a experiencia demonstrou que essa ablação não era necessaria; o hormonio ovariano passa em qualquer caso, como se vê adiante.

\section{EXPERIENCIAS}

6 ratos brancos da mesma ninhada foram tomados para experiencia. Quando attinjiam um peso de 35-37 grs., 3 foram castradas. 25 dias após, foram feitas as parabioses. Os animaes pesavam $75-80$ grs; as normaes já tinham manifestado alguns cyclos estraes, e as castradas permaneciam em diestro desde a castração. Usamos a technica de SAUERBRUCH-HEYDE, (18) colostomia. Abertas as cavidades abdominaes, suturavam-se os animaes em tres planos: peritoneo, musculo e pelle.

A cicatrisação se fez rapidamente e os animaes se conservaram harmonicamente, isto é, sem signaes exteriores que traduzissem anemia ou plethora de um delles. Foram conservados como controle uma normal e uma castrada, livres.

O conteudo vaginal era pesquizado diariamente, com a technica usual; quando havia duvidas sobre a modificação delle, repetia-se 2 a 3 vezes por dia. No quadro figura em cada dia, o que houve de mais caracteristico.

Pode-se lembrar resumidamente que o cyclo sexual dos ratos (LONG e EVANS, 19) pode ser estudado pelo exame do conteudo vaginal. 


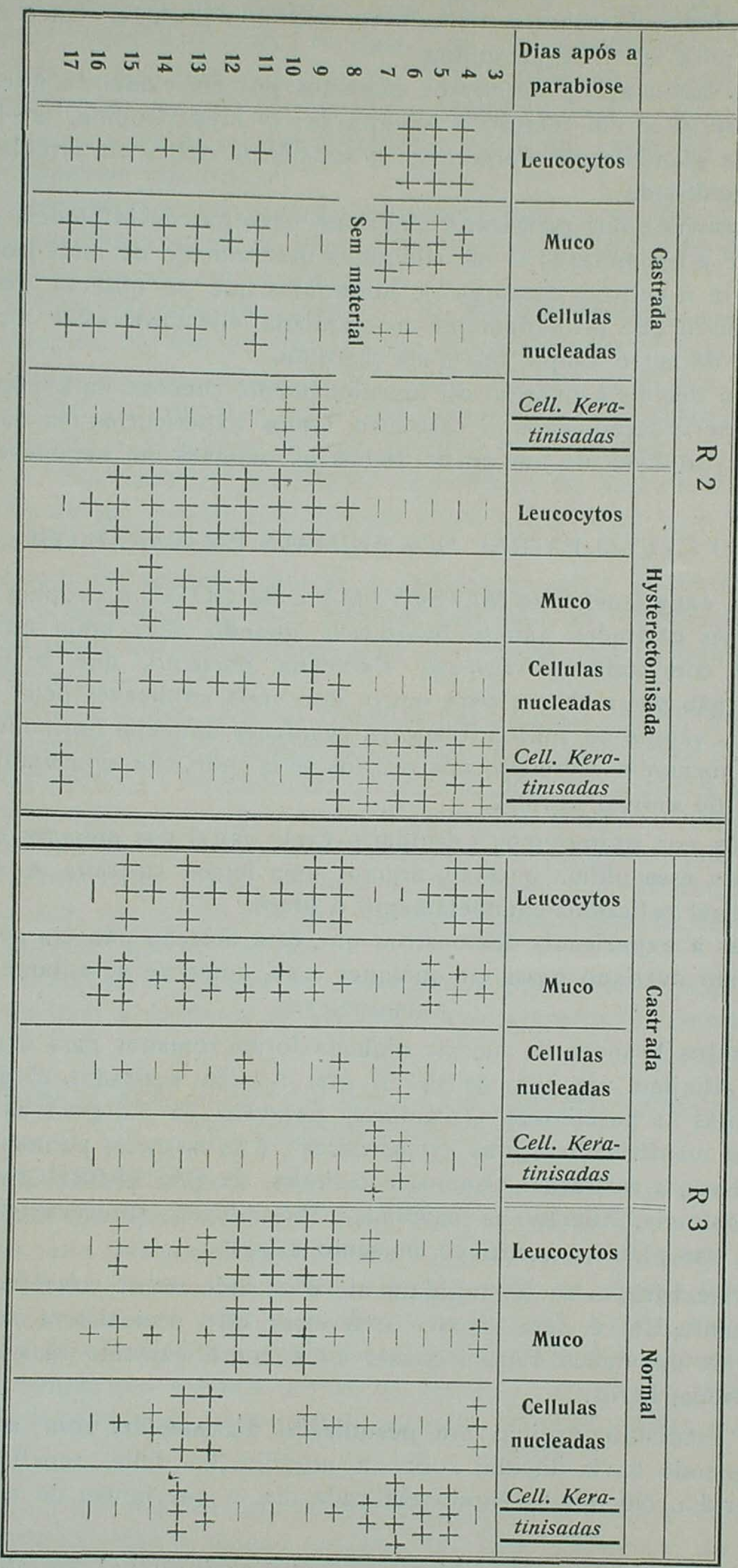




\begin{tabular}{|c|c|c|c|c|c|c|c|c|}
\hline \multirow[b]{2}{*}{$\stackrel{\mathscr{\varpi}}{\sigma}$} & \multicolumn{4}{|c|}{ Controle normal } & \multicolumn{4}{|c|}{ Controle castrada } \\
\hline & 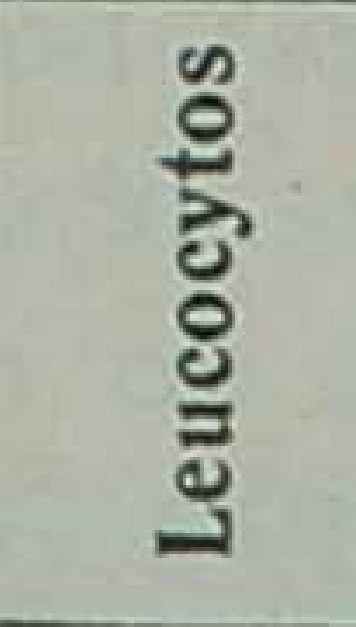 & $\stackrel{8}{\sum}$ & 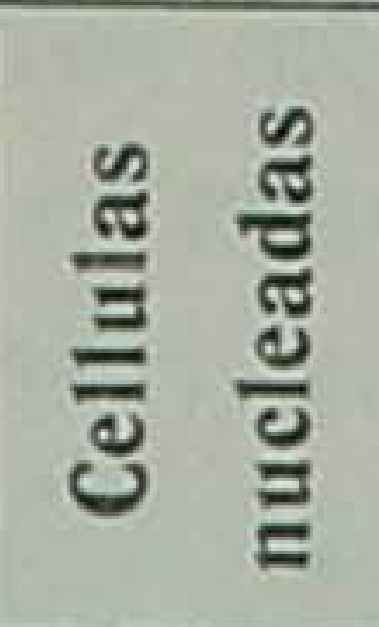 & 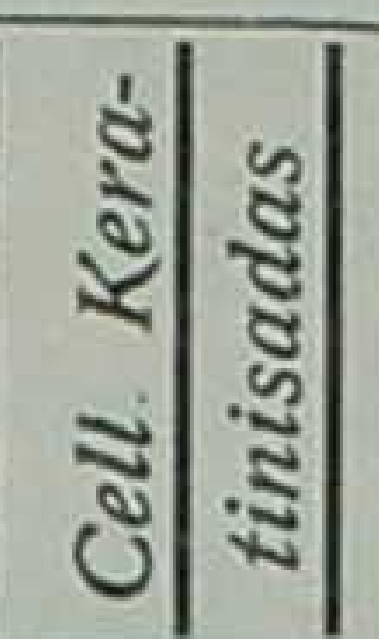 & 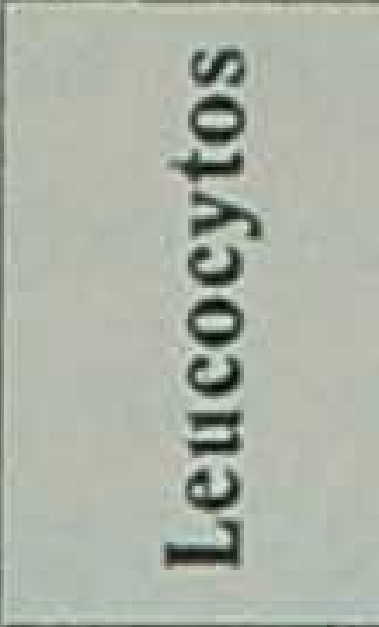 & 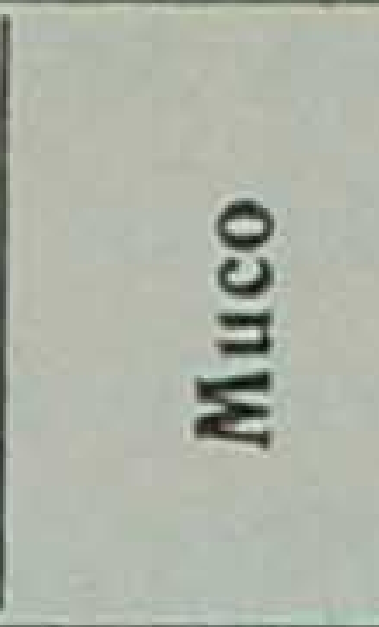 & 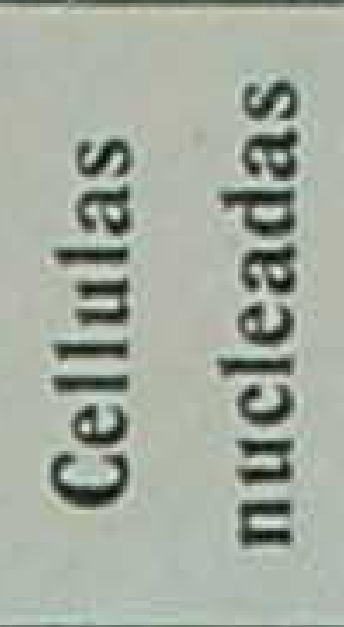 & 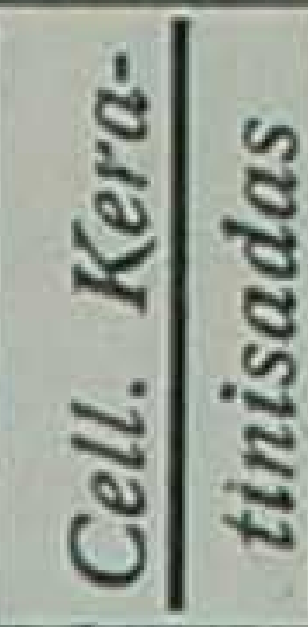 \\
\hline 3 & +++ & ++ & - & - & +++ & ++ & - & - \\
\hline 4 & $+t+$ & + & $t+$ & - & ++ & + & 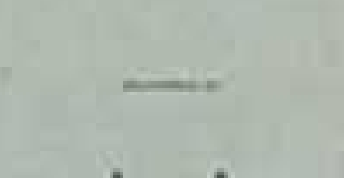 & - \\
\hline 5 & - & - & $++t$ & +++ & ++ & + & ++ & - \\
\hline 6 & - & - & - & +++ & +++ & +++ & + & - \\
\hline 7 & + & +++ & + & - & ++ & +++ & + & - \\
\hline 8 & $+t$ & $+t$ & - & - & \multirow{2}{*}{\multicolumn{4}{|c|}{$\begin{array}{l}\text { Sem material } \\
\text { Sem material }\end{array}$}} \\
\hline 9 & + & $+t$ & + & - & & & & \\
\hline 10 & - & - & - & +++ & + & $+t+$ & - & - \\
\hline 11 & - & - & - & $+t+$ & \multicolumn{4}{|c|}{ Sem material } \\
\hline 12 & +++ & $+t$ & ++ & - & + & $+t+$ & - & - \\
\hline 13 & + & + & - & + & & + & - & - \\
\hline 14 & + & + & ++ & & + & ++ & + & - \\
\hline 15 & - & - & -- & +++ & \multicolumn{4}{|c|}{ Sem materlal } \\
\hline 16 & - & - & - & $t+t$ & + & $+t$ & + & - \\
\hline 17 & + & + & + & - & \multicolumn{4}{|c|}{ Sem material } \\
\hline
\end{tabular}

As variaçðes da mucosa vaginal reflectem a phase do cyclo sexual em que se encontra o animal.

No rato ou no camondongo normal, as phases de actividade, ou estro, são traduzidas pela keratinisação da mucosa; um frottis do conteudo vaginal mostrará grande abundancia de cellulas eosinophilas sem nucleo coravel, e ausencia de leucocytos.

Durante o repouso ou diestro, o frottis mostra leucocytos, muco e eellulas nucleadas, em menor abundancia. A castrada vive invariavelmente em diestro; a introducção de hormonio ovariano, por injecção ou implantações provocará o estado do estro.

O par R2 é formado pela união de uma castrada com uma normal; dessa ultima, foi retirado completamente o utero. de accordo com o raciocinio desenvolvido atraz. R3 é constituido pela parabiose de uma castrada com uma normal. Os controles - normal e castrada - figuram ao lado.

Como se vê, da maneira a mais nitida, as femeas não castradas entram em estro prolongado, pouco depois da união com castrados. No lpar $\mathrm{R} 2$, esse estado de estro foi mantido durante 6 dias; em R3, elle durou cerca de 4 dias.

Facto novo e interessante, o animal castrado tambem manifestou signaes completos de estro, o que significa a passagem de hormonio ovariano proveniente do animal não castrado. Mesmo no animal R3 normal, em que não se fez nenhuma alteração operatoria dos orgãos genitaes, |a passagem de hormonio ovariano para o castrado se fez amplamente. Portanto, o utero e demais receptores não foram obstaculo ao intercambio hormonal. 
Um facto que merece asslgnalado, é a intensidade de keratinisação da mucosa vaginal. Tanto nas parabiosadas castradas como nas não castradas, a massa de cellulas keratinisadas enchia amplamente a vagina, em quantidade que nunca foi por nós observada nos animaes normaes isolados. Note-se é ainda o periodo latente para o estro na femea castrada; assim, em R2 o estro só se manifestou após a terminação do estro na companheira normal; em R3, elle appareceu quando a normal já estava no seu 3o dia.

Emfim, estas experiencias suggerem uma serie de problemas que continuamos a investigar; mas o que já podemos considerar definitiva é a noção da passagem do hormonio ovariano de um animal para outro, em parabiose.

Nesse genero de experiencias, mais do que em qualquer outro, a qualidade tem muito mais importancia do que a quantidade. Nenhuma objecção pode ser feita á serie acima. As femeas castradas o foram cuidadosamente por occasião da operação de parabiose, examinamos os uteros e verificamos a sua atrophia completa. $O$ estro duradouro dos animaes não castrados, nós o interpretamos pela passagem do hormonio pre-hypophysario, que circula em grande abundancia nos animaes castrados.

Posteriermente, publicaremos o estudo macro e microscopico da genitalia interna dos animaes.

\section{A QUESTÃO DA PLURALIDADE DOS HORMONIOS DO LOBO ANTERIOR.}

Alem do hormonio ou hormonios da hypophyse anterior, que exercem um estimulo sobre as gonadas, EVANS e LONG (20) extrahiram do orgão substancias que exercem acção inhibidora sobre o estro das ratas.

Relativamente aos hormonios estimulantes que estudamos atraz, ha a assignalar, como effeitos os seguintes, nos animaes femeas, obtidos pelos varios autores citados:

10 Desenvolvimento dos folliculos ovarianos.

20 Formação de corpora lutea atresica.

3o Formação de corpora lutea, ap ós ovulação.

Com a implantação de lobo anterior da hypophyse, os effeitos 1 e 3 predominaram. Pode-se, em determinados casos, provocar apenas o desenvolvimento dos folliculos, como é exemplo a figura 2.

Usando a urina de mulher gravida como fonte de material, encontrase no camondongo, um aspecto macroscopico especial, que ZONDEK e ASCHHEIM denominaram "Blut punkte", isto é, pontos hemorrhagicos que se distinguem muito bem, a olho nú na superficie dos ovarios, e que são um elementos para seu bello processo de diagnostico da gravidez.

Corn as implantaçð̃es, nunca obtivemos "Blut punkte", donde concluimos que pelo menos elles não são frequentes como no caso da urina, o que tambem observou ENGLE (21).

A formação de corpora lutea atresica é a forma mais constante, quando se injecta urina. A reacção 1, de ZONDEK e ASCHHEIM, isto é, injecção de urina. 


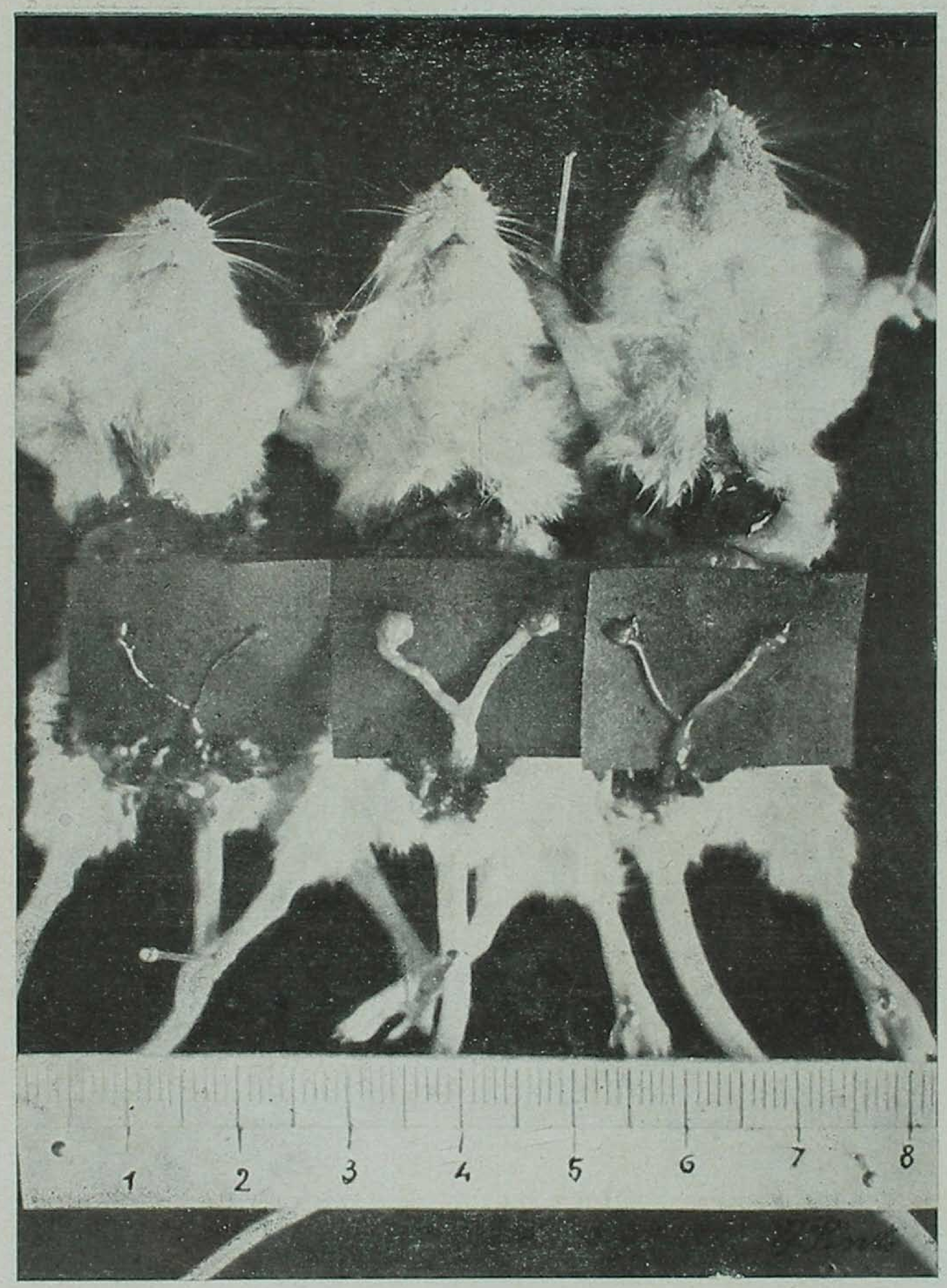

C

I

C

Fig. 1-...Puberdade precoce de camondongo infantil de $7,5 \mathrm{gr}$., aos lados dois controles. O animal foi implantado com 2 lobos anteriores de hypophyse de coelho. I-implantado. C-controles.

Fig. 1-Precocious sexual maturity, following 2 implantations of anterior pituitary of rabbit. I-7,5 gr. mous, implanted. C-Untreated controls. 


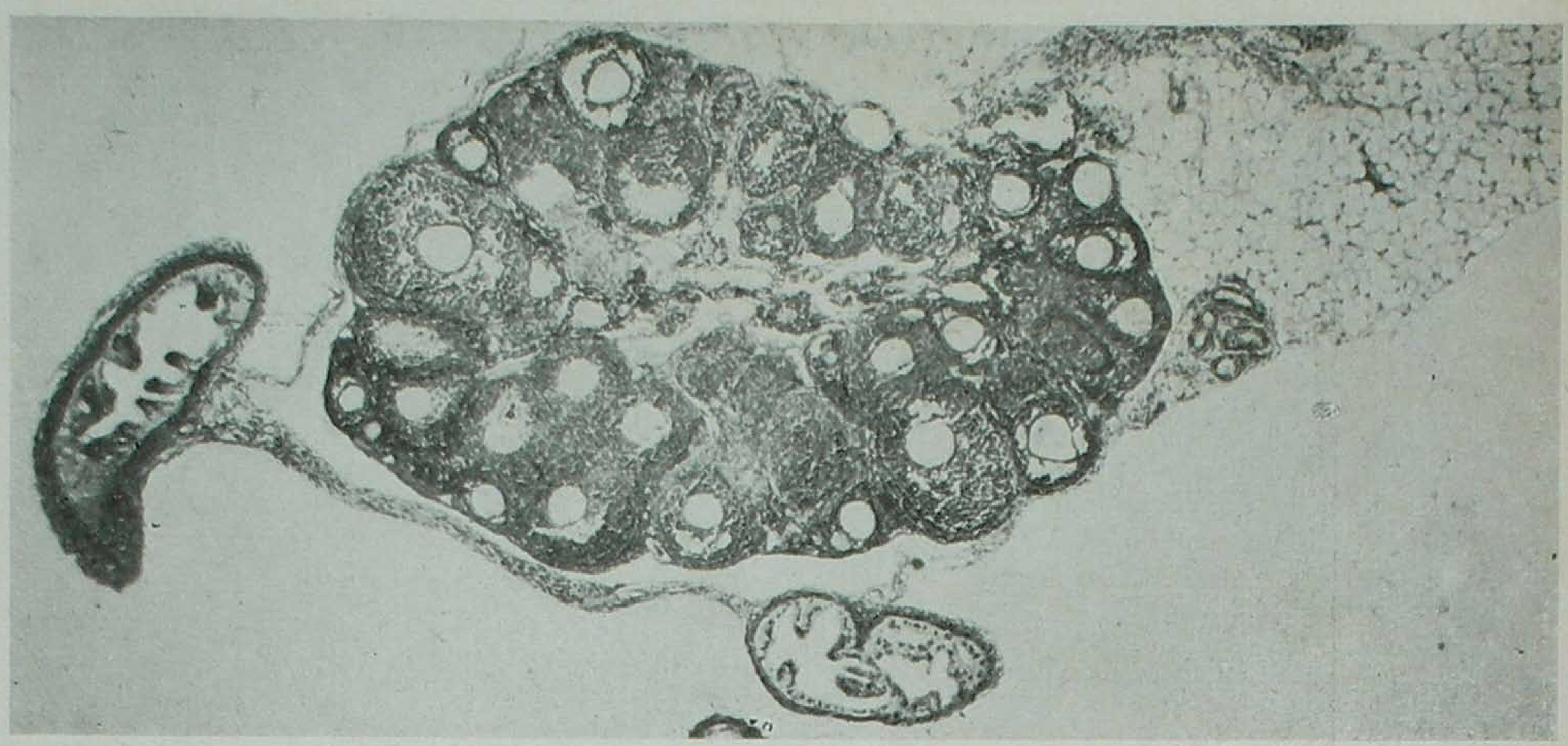

Fig. 2 - Corte de ovario e trompa de 1 m camondongo branco infantil de 7,5 grs.

Fig. 2-Cross sectiou of ovary and tuba of an 7,5 grs., immature mouse.

$1 \times 45$.

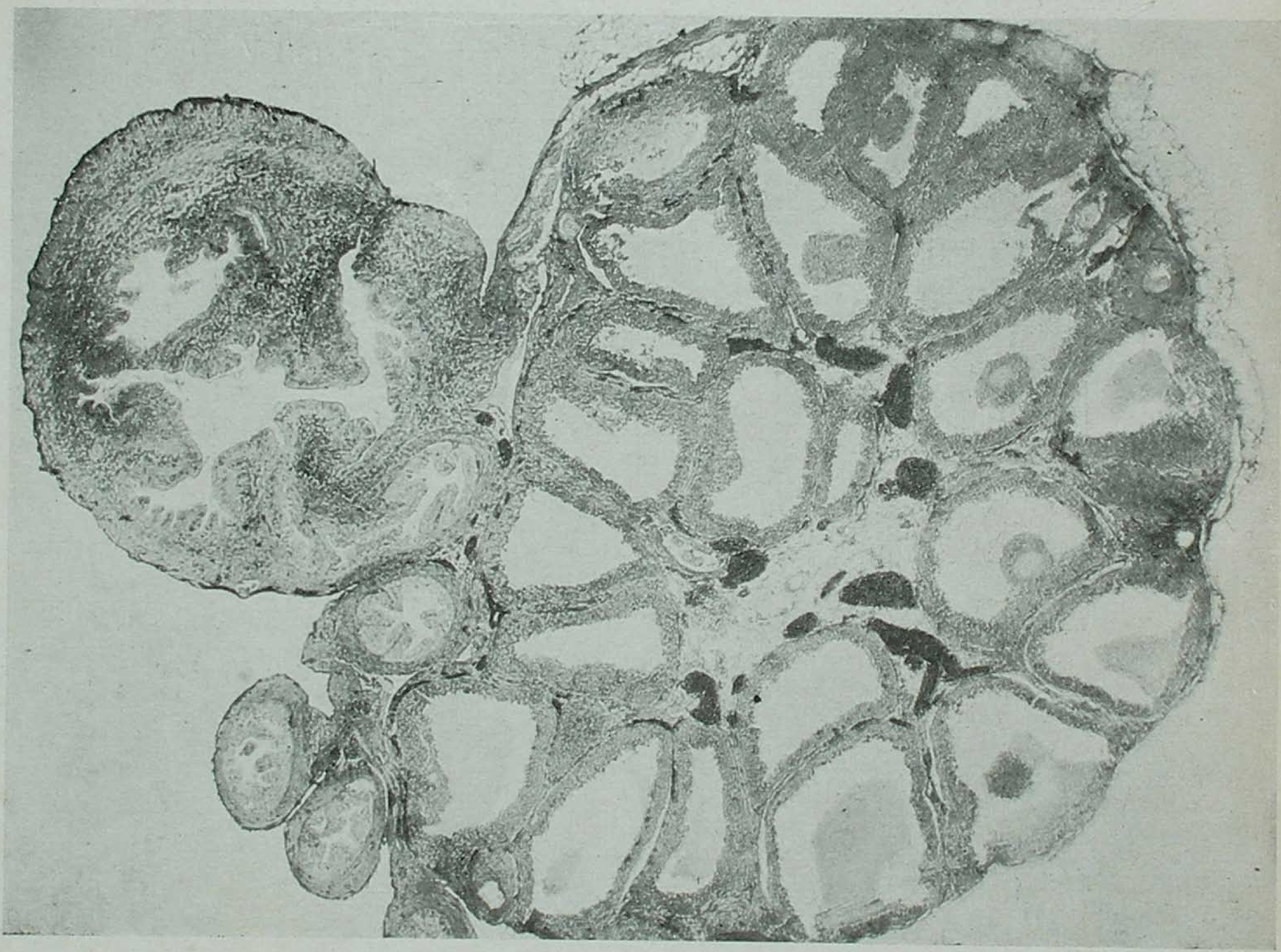

Fig. 3-Corte do ovario do animal implantado, visto na fig. 1.

Fig. 3-Cross section of the ovary, of implanted mouse shown in fig. 1. 


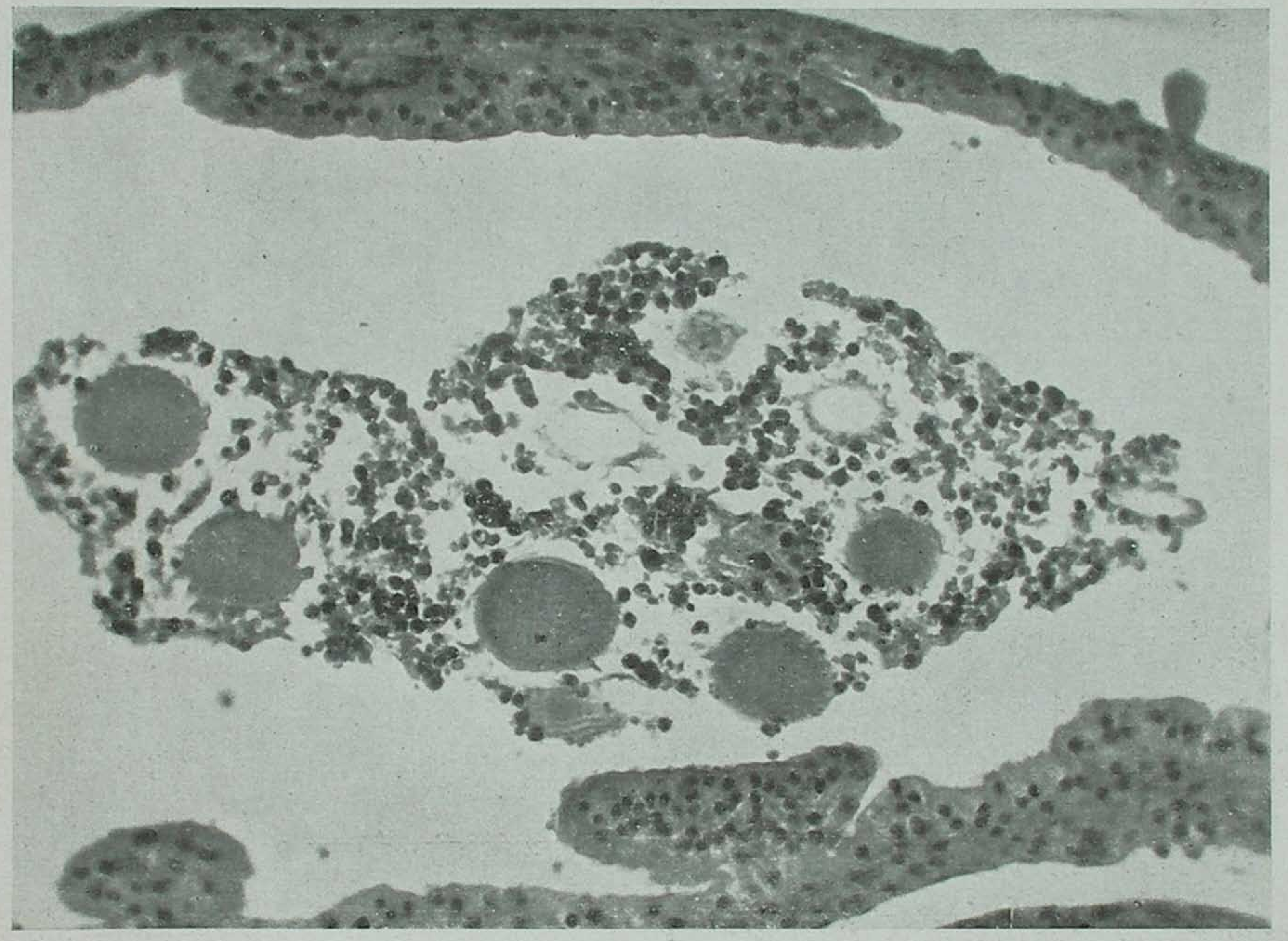

Fig. 4-Ovulos na trompa de um camondongo infantil de 7,5 grs., em puberdade precoce, 100 horas após a implantação de 1 lobo anterior de hypophyse de coelho.

Fig 4-Tubal ova; precocious sexual maturity, 100 hours aften one single implantation of 1 anterior pituitary of rabbit, in a 7,5 grs. mous. 


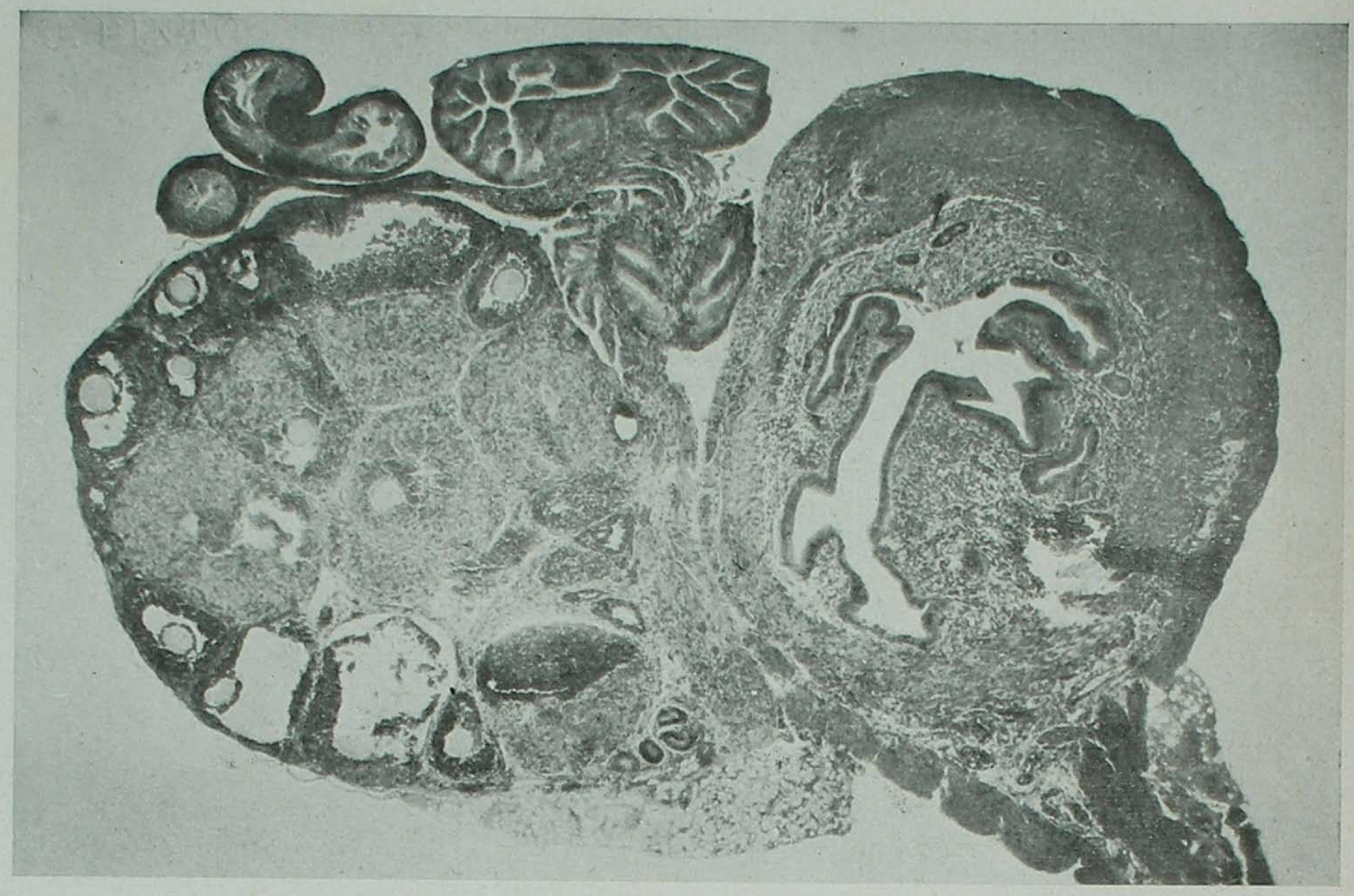

Fig. 5-Desenvolvimento dos folliculos, formação de corpora lutea atresica, folliculo hemorrhagico, após injecção de urina gravidica, dentro de um praso de 100 horas. Camondongo de 7 grs.

Fig. 5-Follicular development, corpora lutea atresica, hemorrhagic follicle, after injection of pregnant woman urine, in a 7 grs. mous. These changes are obtained within 100 hours $1 \times 45$.

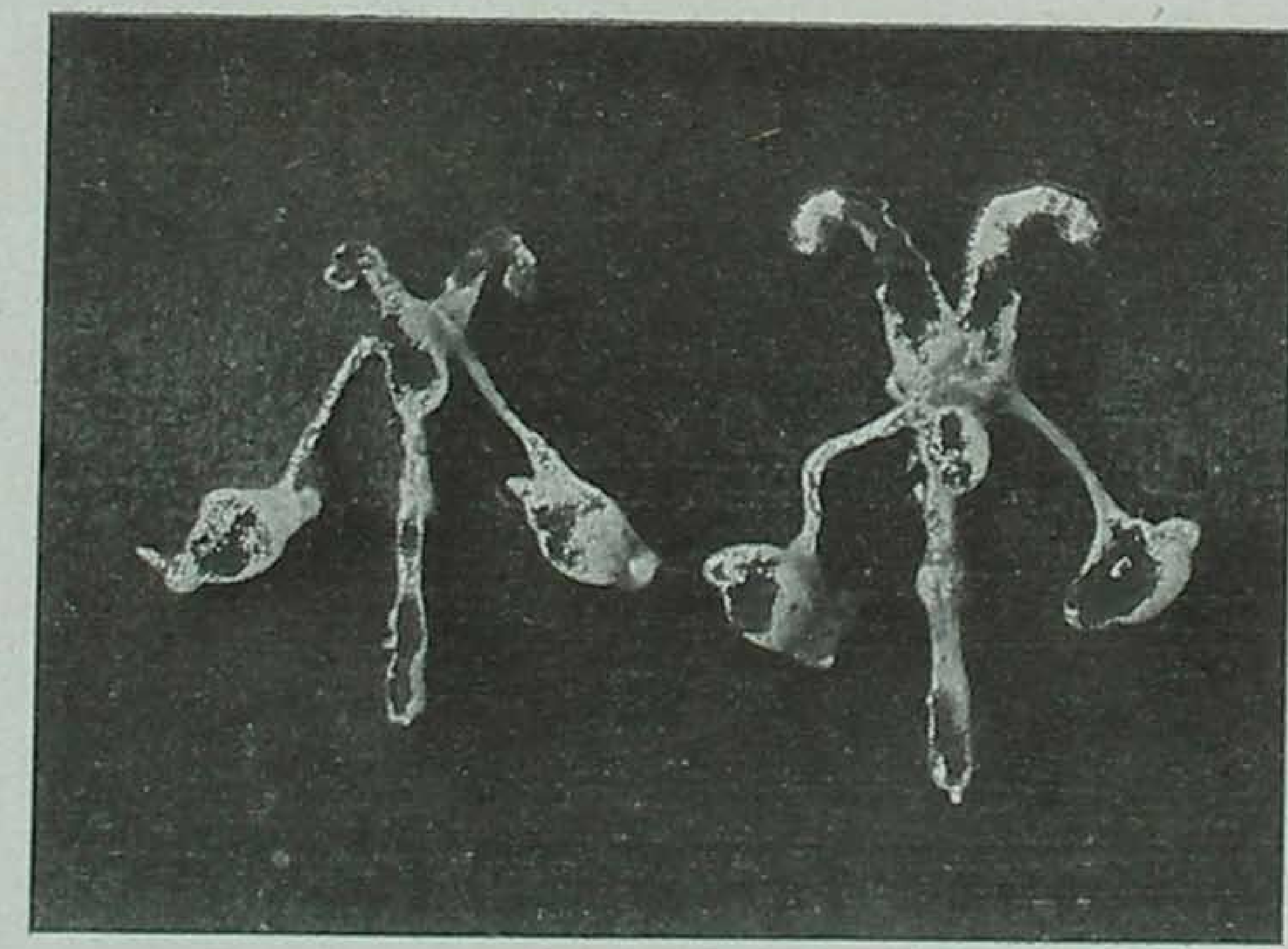

C I

Fig. 6-Puberdade precoce provocada por 3 implantações de hypophyse anterior de coelho, em camondongo de 9 grs. Notar o desenvolvimento do penis e vesiculas seminaes. C-controle.

Fig. 6-Precocious sexual maturation, followiog 3 ant. hyp. of rabbit, implantations, in a 9 grs., male mous. Development of penis and seminal vesicles. C-control. 
Nenhum argumento serio se pode invocar, para negar a identidade entre os hormonio hypophysarios eli minados na gravidez e os produzidos pela hypophyse.

Ha entretanto evidencias de variações quantitativas e qualitativas desses hormonios, conforme as situações physiologicas ou experimentaes.

Notadamente o fa ctor atresiante domina na urina gravidica.

A elaboração de mais de um hormonio pelo lobo anterior é uma possibilidade perfeitamente rasoavel.

Até certo ponto, o antagonismo entre a ação estimulante do desenvolvimento follicular e a acção lıteinisante, dão idea de um mechanismo muito bem adaptado para a regulação do cyclo ovarianno.

ASCHHEIM (22), recentemente, tende a admittir a pelo menos dualidade do hormonio, e assim tambem BRÜHL (23).

Do que observamos e pesquisamos, somos levados a concordar com esse ponto de vista; mas por emquanto nada podemos dizer de definitivo, e pensamos dar em breve conclusões mais seguras,

(Laboratorio de Physiologia do Instituto Oswaldo Cruz).

BIBLIOGRA PHIA

Ver no final da traducção ingleza. 